\title{
Cross-Sectional and Longitudinal Associations of BMI with Socioeconomic Characteristics
}

\author{
Mahasin S. Mujahid,* Ana V. Diez Roux, * Luisa N. Borrell, $\dagger$ and F. Javier Nieto
}

\begin{abstract}
MAHASIN S. MUJAHID, DIEZ ROUX, ANA V., BORRELL, LUISA N., AND NIETO, F. JAVIER. Crosssectional and longitudinal associations of BMI with socioeconomic characteristics.Obes Res. 2005;13:1412-1421.

Objective: To examine cross sectional and longitudinal associations of socioeconomic position and neighborhood environments with BMI in a middle-aged and bi-ethnic cohort.

Research Methods and Procedures: Analyses were based on 13,167 subjects (45 to 64 years) who participated in the Atherosclerosis Risk in Communities Study, a populationbased study. Census block groups were used as proxies for neighborhoods and were characterized using a summary socioeconomic score. BMI was measured at baseline and at three follow-up visits over a 9-year period.

Results: Individual and neighborhood socioeconomic characteristics were independently and inversely associated with BMI at baseline in women [mean difference in kilograms per meter squared per unit increase in socioeconomic category (SE) for white and black women respectively; -1.56 $(0.14),-1.59(0.19)$ for education; $-1.07(0.10),-1.18$ (0.18) for income; and $-1.04(0.09),-0.77(0.18)$ for neighborhood characteristics]. Results for men were not as consistent. Baseline BMI was negatively associated with income in white men but was positively associated with education, income, and neighborhood characteristics in black men. BMI increased over time regardless of gender or race and in most age groups. In whites, there were no consistently patterned differences in longitudinal trends in BMI by individual or neighborhood socioeconomic charac-
\end{abstract}

Received for review November 3, 2004

Accepted in final form May 23, 2005.

The costs of publication of this article were defrayed, in part, by the payment of page charges. This article must, therefore, be hereby marked "advertisement" in accordance with 18 U.S.C. Section 1734 solely to indicate this fact.

*Department of Epidemiology, University of Michigan, Ann Arbor, Michigan; $†$ Department of Epidemiology, Columbia University, New York; and Department of Population Health, University of Wisconsin, Madison, Wisconsin.

Address correspondence to Ana V. Diez Roux, Department of Epidemiology, 1214 South University, Ann Arbor MI 48103.

E-mail: adiezrou@umich.edu

Copyright (C) 2005 NAASO teristics. However, in blacks, there was some evidence of greater increases in the higher socioeconomic status groups. Discussion: Socioeconomic factors are inversely associated with BMI in middle-aged women, possibly reflecting socially patterned exposures occurring in childhood and adolescence. However, recent increases over time in BMI are either not clearly patterned by socioeconomic factors or are greater in the higher socioeconomic status groups.

Key words: BMI, socioeconomic status, race, ethnicity, neighborhoods

\section{Introduction}

Obesity has emerged as a major public health problem in recent years (1-5). Data from the National Health and Nutrition Examination Surveys show that the prevalence of obesity (BMI $\geq 30 \mathrm{~kg} / \mathrm{m}^{2}$ ) in the United States has increased from $14.5 \%$ in 1971 to 1974 to $30.9 \%$ in 1999 to 2000 (6). This increase appears to have occurred in both men and women and across all ages and racial/ethnic groups $(2,3,6)$. The reasons for the increase remain a subject of debate. A variety of explanations have been proposed, including changes in diet and physical activity habits $(6,7)$.

Although many studies and surveys have examined secular trends in obesity, weight, or BMI, factors associated with differential increases within the U.S. population have been infrequently examined. In addition, most reports of changes in BMI over time involve the analysis of repeat cross-sectional surveys $(4-6,8)$. Longitudinal studies of weight change, which allow simultaneous examination of aging-related changes and secular trends are rare. The identification of factors associated with greater increases in BMI over time would contribute to our understanding of the causes of weight gain and allow the targeting of preventive interventions.

There is abundant evidence of the socioeconomic patterning of cardiovascular risk, with higher incidence of cardiovascular disease and higher prevalence of cardiovascular risk factors in the lower socioeconomic groups (9). Recent data also suggest that neighborhood socioeconomic disad- 
vantage is associated with the incidence of coronary heart disease $(10,11)$. It has been hypothesized that low socioeconomic position and living in deprived neighborhoods may expose persons to environments less conducive to healthy eating and physical activity, which can lead to increases in weight gain over time $(9,12)$. It has also been hypothesized that there are stress-related mechanisms linking low socioeconomic status (SES) ${ }^{1}$ to weight gain (13). However, the extent to which personal and neighborhood socioeconomic characteristics are associated with greater weight gain over time has not been established. Using data from a longitudinal study of atherosclerosis, we examined the cross-sectional and longitudinal associations of education, family income, and neighborhood environments with BMI in a middle-aged cohort (45 to 64 years old). We hypothesized that BMI would be inversely associated with socioeconomic factors in cross-sectional analyses and that increases over time in BMI would be greater among persons of low socioeconomic position and in those living in disadvantaged neighborhoods.

\section{Research Methods and Procedures Study population and study variables}

The Atherosclerosis Risk in Communities (ARIC) Study is a prospective investigation of atherosclerosis in four U.S. communities (Forsyth County, NC; Jackson, MS; the northwestern suburbs of Minneapolis, MN; and Washington County, MD) (14). The ARIC cohort was composed of 15,792 persons aged 45 to 64 years at the time of the baseline interview, selected by random sampling in the four communities (ARIC Investigators 1989). Two of the samples (Washington County and Minneapolis suburbs) are virtually all white. The Forsyth County sample is $85 \%$ white. The Jackson, MS sample is entirely African American. The baseline examination of the ARIC cohort (Visit 1) took place between 1987 and 1989. Follow-up exams were carried out $\sim 3$ years later (1990 to 1992), 6 years later (1993 to 1995), and 9 years later (1996 to 1999). Retention rates were $93 \%, 87 \%$, and $81 \%$ at the first, second, and third follow-up exams, respectively.

Weight measurements were obtained at baseline and each follow-up visit using standardized procedures. Height measurements were also obtained at baseline and follow-up visits excluding the first follow-up. Height information at baseline was used as an estimate for a participant's height at the first follow-up. BMI was calculated as weight in kilograms divided by height in meters squared. Information on neighborhood socioeconomic characteristics was obtained from the 1990 U.S. Census. Participants were linked to their neighborhood of residence using their baseline home ad-

\footnotetext{
${ }^{1}$ Nonstandard abbreviation: SES, socioeconomic status; ARIC, Atherosclerosis Risk in Communities.
}

dress. Census-defined block groups (subdivisions of census tracts) were used as proxies for neighborhoods. A summary score was used to characterize the neighborhood socioeconomic environment. The variables used in the construction of the score were selected based on prior factor analyses of census block group data (15). Six census variables representing the dimensions of wealth/income (log median household income, log median value of housing units, and percentage of households receiving interest, dividend, or net rental income), education (percentage of adults with complete high school, percentage of adults with complete college), and occupation (percentage of persons in executive, managerial, or professional specialty occupations) were combined into the neighborhood summary score. For each variable, a $z$ score for each block group was estimated by subtracting the overall mean (across all block groups in the sample) and dividing by the SD. The $z$ score reflects the deviation of the value from the mean in SD units. The neighborhood summary score was constructed by summing the $z$ scores for each of the six variables. Neighborhood scores for block groups in the sample ranged from -11.3 to 14.5 , with increasing score signifying increasing neighborhood advantage. Because there was relatively little overlap in the types of neighborhoods in which whites and blacks lived, neighborhood score was categorized into race-specific tertiles. The cohort was quite stable over the follow-up period, and only $18 \%$ of participants had moved 6 years after the baseline examination.

Information on individual-level measures of education and income was obtained from the baseline interview of the ARIC study. Participants were asked to report the highest grade or year of school completed and to select their total combined family income from a list of eight categories (under $\$ 5000, \$ 5000$ to $\$ 7999, \$ 8000$ to $\$ 11,999, \$ 12,000$ to $\$ 15,999, \$ 16,000$ to $\$ 24,999, \$ 25,000$ to $\$ 34,999$, $\$ 35,000$ to $\$ 49,999$, and $\$ 50,000$ or more) (16). Education was categorized into three levels (less than high school, completed high school, and completed college or more). Three income categories containing as close as possible to $33 \%$ of the sample were created in each race group (\$0 to $\$ 24,999, \$ 25,000$ to $\$ 49,999$, and $\$ 50,000$ or more in whites and $\$ 0$ to $\$ 11,999 ; \$ 12,000$ to $\$ 24,999$, and $\$ 25,000$ or more in blacks). Race-specific income categories were used due to large differences in the distribution of income by race. Information on smoking status (current, former, and never smoker), self-reported history of cancer, and selfreported health (excellent, good, fair, and poor) was collected at baseline and each follow-up visit.

Of the 15,792 ARIC participants at the baseline examination, 91\% $(14,351)$ were successfully geocoded to the block group level, and 14,158 matched to non-excluded census areas (population $>100$, housing units $>30,<33 \%$ of inhabitants living in group quarters, and measures available for all neighborhood score components). Of these, 43 
reported being of racial/ethnic groups other than African American or white and were excluded from these analyses. Because small numbers made race- and center-specific analyses unreliable, an additional 55 African-American participants living in the Minneapolis suburbs or Washington County were also excluded. Participants were also excluded if information was missing on individual-level education and/or occupation $(n=56)$. Of participants with no information on income, $\sim 6 \%$ were also excluded, leaving a total of 13,167 participants for analysis. Of these $13,167,71 \%$ had BMI information for all four visits, $12 \%$ had information for three of the four visits, $10 \%$ had information for two of the four visits, and $7 \%$ had information for one visit. The final sample was distributed over 594 block groups, with a median of 16 participants per block group (range, 1 to 140 participants).

\section{Statistical methods}

All analyses were race and gender specific. Graphical methods (17) were initially used to explore patterns in BMI by age and calendar time. Cross-sectional differences and longitudinal changes in BMI by education, income, and neighborhood characteristics before and after adjustment for covariates (age, smoking, history of cancer, and self-reported health) were estimated using mixed models (PROC MIXED SAS version 8.2; SAS Institute Inc., Cary, NC). Education, income, and neighborhood characteristics were initially examined in separate models and then together in the same model to estimate their independent effects. Models regressed BMI at each examination on baseline age, time since baseline, baseline SES level, and the interactions between time and baseline SES. The basic model fitted is shown below:

$$
\begin{aligned}
& Y i j=\beta_{0}+\beta_{1}\left(\text { age }_{i 0}\right)+\beta_{2}\left(\text { time }_{i j}\right)+\beta_{3}\left(\text { age }_{i 0} \times \text { time }_{i j}\right) \\
& +\beta_{4}\left(\text { SES }_{i 0}\right)+\beta_{5}\left(\text { SES }_{i 0} \times \text { time }_{i j}\right)+U_{i 0}+U_{i 1} \times \text { time }_{i j}+e_{i j}
\end{aligned}
$$

where $\mathrm{Y}_{\mathrm{ij}}$ is $\mathrm{BMI}$ for person $\mathrm{i}$ at time $\mathrm{j}$, age $\mathrm{i}_{\mathrm{i}}$ is age at baseline, time $_{i j}$ is time since baseline for person $i$ at visit $j$, $\mathrm{SES}_{\mathrm{i} 0}$ is the socioeconomic indicator at baseline, and $\mathrm{U}_{\mathrm{i} 0}$ is a random intercept for person $\mathrm{i}$ and $\mathrm{U}_{1 \mathrm{j}}$ is a random time slope for person $i$.

The interaction terms between time and baseline SES level were included to allow longitudinal change estimates to differ by levels of baseline SES and to allow statistical testing of these differences. A time-by-baseline age interaction was included in all models because changes over time were found to differ significantly by baseline age, with greater increases over time in those younger at baseline. Interactions between time and baseline age were statistically significant with $p<0.001$ for all models. Trends across the three categories of education, income, or neighborhood score studied were tested by including the variable as an ordinal covariate with scores of 1 to 3 . Covariate-adjusted models also included current smoking, self-reported health, and history of cancer as time-dependent variables for each visit. All models allowed both the intercept and time effect to vary randomly across persons because it significantly improved the model fit. Patterns for BMI and weight were virtually identical, so only results for BMI are reported. All results were similar when analyses were restricted to persons with BMI measures at all four visits. Results were also robust when models were stratified by baseline BMI. Analyses were repeated in persons under 55 years of age and 55 years of age and older at baseline. Although increases in BMI were smaller in the older age groups, patterns by SES were very similar, so only pooled results adjusted to age 55 are shown.

In our sample, $29 \%$ of participants had missing information for at least one visit. To investigate the possible impact of missing values on our estimates, we examined the distributions of age, race, gender, education, income, and neighborhood characteristics by the number of completed visits. In addition, to assess whether drop-outs (due to death or loss to follow-up) had different BMI trajectories than persons who remained in the study, we estimated longitudinal trends separately for participants with three or more visits and for participants with fewer than three visits and compared the results.

\section{Results}

Table 1 shows characteristics of the study sample by race and gender. Black study participants were generally of lower education and income and tended to live in more disadvantaged neighborhoods than white participants. Mean BMI increased over follow-up in both racial groups regardless of gender. Overall, the largest increases over time were observed in white women, and the smallest increases were observed in black men, with mean increases of 0.92 and $0.34 \mathrm{~kg} / \mathrm{m}^{2}$, respectively, over a 5 -year period.

As reported elsewhere (18), BMI generally increased over the follow-up regardless of baseline age. Increases over time tended to be more pronounced for younger birth cohorts (data not shown). Table 2 shows the mean BMI at baseline and longitudinal changes in BMI over the follow-up period, by race, gender, education, family income, and neighborhood characteristics. The estimates shown are adjusted to age 55 years. Baseline BMI was inversely associated with education and income in white men, white women, and black women, although education and income differences in white men were small (mean difference per unit increase in category $=-0.20,-1.56$, and $-1.59 \mathrm{~kg} / \mathrm{m}^{2}$ for education and $-0.15,-1.07$, and $-1.18 \mathrm{~kg} / \mathrm{m}^{2}$ for income for white men, white women, and black women, respectively). In contrast, baseline BMI was positively associated with education and income in black men (mean difference per unit increase in category $=0.36$ and 0.73 $\mathrm{kg} / \mathrm{m}^{2}$ for education and income, respectively). Mean BMI 
Table 1. Characteristics of study participants by race and gender at baseline (1987 to 1989) and changes in BMI over 9 years, the ARIC Study

\begin{tabular}{|c|c|c|c|c|}
\hline & \multicolumn{2}{|c|}{ Whites } & \multicolumn{2}{|c|}{ African Americans } \\
\hline & $\begin{array}{c}\text { Men } \\
(n=4641)\end{array}$ & $\begin{array}{c}\text { Women } \\
(n=5115)\end{array}$ & $\begin{array}{c}\text { Men } \\
(n=1275)\end{array}$ & $\begin{array}{c}\text { Women } \\
(n=2136)\end{array}$ \\
\hline Mean age at baseline (SD) & $54.9(5.7)$ & $54.0(5.7)$ & $53.9(6.0)$ & $53.3(5.7)$ \\
\hline \multicolumn{5}{|l|}{ Income (\% distribution) } \\
\hline$<\$ 12,000$ & 4.1 & 9.0 & 30.0 & 46.5 \\
\hline$\$ 12,000$ to $\$ 24,999$ & 16.4 & 22.2 & 31.3 & 29.0 \\
\hline$\$ 25,000$ to $\$ 34,999$ & 19.8 & 19.8 & 14.7 & 11.3 \\
\hline$\$ 35,000$ to $\$ 49,999$ & 24.6 & 21.8 & 13.4 & 8.2 \\
\hline$\geq \$ 50,000$ & 35.1 & 27.2 & 10.6 & 5.0 \\
\hline \multicolumn{5}{|l|}{ Education ( $\%$ distribution) } \\
\hline Incomplete high school & 17.8 & 15.5 & 42.6 & 40.3 \\
\hline Complete high school or GED & 39.2 & 51.4 & 26.3 & 29.2 \\
\hline 1 to 3 years college & 15.1 & 17.6 & 11.0 & 9.1 \\
\hline 4 years college & 15.1 & 9.8 & 7.6 & 8.1 \\
\hline Graduate school & 12.8 & 5.7 & 12.5 & 13.3 \\
\hline \multicolumn{5}{|l|}{ Neighborhood score } \\
\hline Median (25th,75th) & $2.2(-0.05,4.8)$ & $2.1(-0.1,4.5)$ & $-3.5(-6.4,-1.0)$ & $-5.1(-6.5,-1.8)$ \\
\hline \multicolumn{5}{|l|}{ Mean BMI $\left(\mathrm{kg} / \mathrm{m}^{2}\right)(\mathrm{SD})[\mathrm{n}]$} \\
\hline Baseline & $27.4(4.0)[4640]$ & $26.6(5.5)[5113]$ & $27.6(4.9)[1272]$ & $30.8(6.5)[2135]$ \\
\hline First follow-up & $27.6(4.1)[4335]$ & $27.0(5.6)[4812]$ & $28.0(4.8)[1029]$ & $31.1(6.7)[1807]$ \\
\hline Second follow-up & $28.1(4.4)[3955]$ & $27.8(5.8)[4461]$ & $28.3(5.1)[860]$ & $31.5(6.8)[1543]$ \\
\hline Third follow-up & $28.4(4.4)[3583]$ & $28.2(5.9)[4086]$ & $28.5(5.1)[753]$ & $31.8(6.9)[1378]$ \\
\hline Mean 5-year change in BMI (SD) & $0.57(0.02)$ & $0.92(0.02)$ & $0.34(0.04)$ & $0.68(0.04)$ \\
\hline
\end{tabular}

at baseline was also inversely associated with neighborhood scores in white and black women (mean difference per unit increase in category $=-1.04$ and $-0.77 \mathrm{~kg} / \mathrm{m}^{2}$, respectively), but no clear trends were observed in white men. Mean BMI was positively associated with neighborhood scores in black men, although the differences were small (mean difference per unit increase in category $=0.24 \mathrm{~kg}$ / $\mathrm{m}^{2}$ ). Associations of neighborhood score with baseline BMI, in women, were reduced and remained statistically significant for white women after additional adjustment for education and income (adjusted mean differences per unit increase in category $=-0.64$ and $-0.25 \mathrm{~kg} / \mathrm{m}^{2}$ for white and black women, respectively) (data not shown).

Mean BMI increased over follow-up in all education, income, and neighborhood categories and in all race and sex groups (Table 2). In whites, there were no clear patterns in trends over time by education, income, or neighborhood characteristics. The only statistically significant trend was observed for income in white women, but the direction of the association was contrary to that expected (income was positively associated with BMI increase). In black women, both education and income were positively associated with BMI increases. Similar patterns were observed for neighborhood score in black women and for all SES indicators in black men, although tests for trend were not statistically significant. The patterns described above did not change substantially for education, income, or neighborhood score after controlling for all other available socioeconomic variables at baseline and their interactions with time (data not shown). Additional controls for health, cancer, and smoking status at each visit as time-dependent covariates also did not substantially modify the patterns observed (data not shown). Patterns were similar when analyses were stratified by age at baseline ( $<55$ vs. 55 years and over).

As shown in Table 3, there is evidence that the number of repeat BMI measures available for analysis differed by race and socioeconomic indicators. Blacks, persons in the lower income categories, and more disadvantaged neighborhoods tended to be overrepresented in the portion of the sample with only one or two BMI measures (compared with three or four measures). However, estimates of changes over time were similar for persons with two measures and for those 


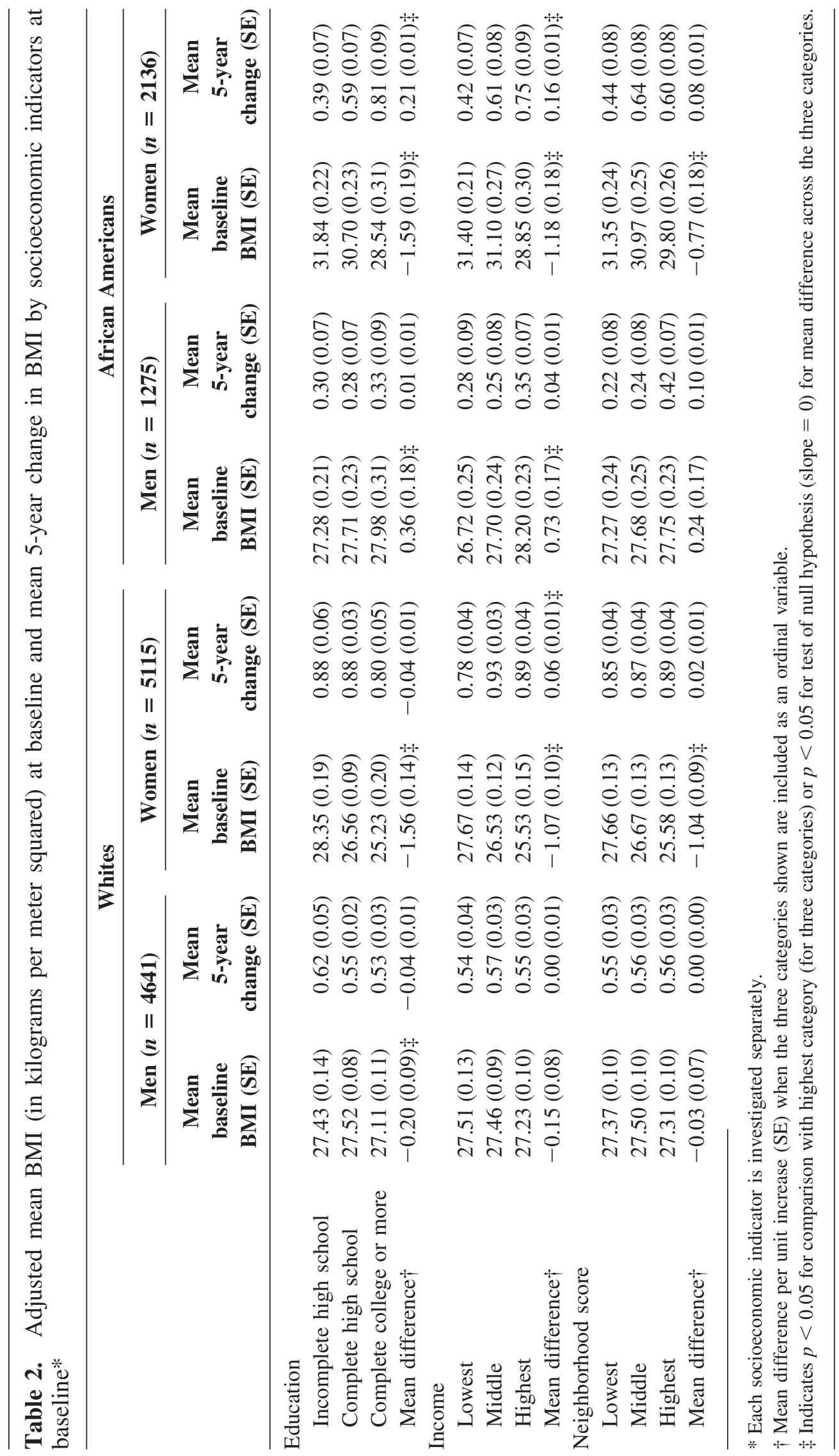


Table 3. Distribution of baseline variables by number of study visits for which BMI is available

\begin{tabular}{|c|c|c|c|c|}
\hline & $\begin{array}{l}\text { One visit } \\
(N=918)\end{array}$ & $\begin{array}{l}\text { Two visits } \\
(N=1270)\end{array}$ & $\begin{array}{l}\text { Three visits } \\
(N=1612)\end{array}$ & $\begin{array}{l}\text { Four visits } \\
(N=9367)\end{array}$ \\
\hline Mean age at baseline (SD) & $55.3(6.1)$ & $54.7(5.9)$ & $54.4(5.8)$ & $54.0(5.7)$ \\
\hline \multicolumn{5}{|l|}{ Race (\% distribution) } \\
\hline White & 54.6 & 59.6 & 63.4 & 79.8 \\
\hline African American & 45.4 & 40.4 & 36.6 & 20.2 \\
\hline \multicolumn{5}{|l|}{ Gender (\% distribution) } \\
\hline Male & 48.9 & 45.3 & 45.8 & 44.3 \\
\hline Female & 51.1 & 54.7 & 54.2 & 55.7 \\
\hline \multicolumn{5}{|l|}{ Income (\% distribution) } \\
\hline$<\$ 12,000$ & 32.7 & 26.6 & 22.6 & 10.9 \\
\hline$\$ 12,000$ to $\$ 24,999$ & 27.5 & 24.0 & 25.4 & 22.8 \\
\hline$\$ 25,000$ to $\$ 34,999$ & 13.2 & 16.8 & 15.5 & 18.9 \\
\hline$\$ 35,999$ to $\$ 49,999$ & 12.6 & 15.8 & 16.7 & 21.6 \\
\hline$\geq \$ 50,000$ & 14.0 & 16.8 & 19.8 & 27.8 \\
\hline \multicolumn{5}{|l|}{ Education (\% distribution) } \\
\hline Incomplete high school & 44.5 & 36.2 & 31.0 & 17.7 \\
\hline Complete high school or GED & 33.9 & 37.4 & 37.9 & 42.8 \\
\hline 1 to 3 years of college & 11.0 & 12.1 & 14.3 & 15.5 \\
\hline 4 years college & 5.7 & 6.8 & 9.1 & 12.6 \\
\hline Graduate school & 4.9 & 7.5 & 7.7 & 11.4 \\
\hline \multicolumn{5}{|l|}{ Neighborhood score } \\
\hline Median (25th, 75th) & $-1.2(-5.4,2.2)$ & $-0.2(-4.0,2.7)$ & $-0.04(-3.6,2.9)$ & $1.49(-1.1,4.0)$ \\
\hline Mean BMI at baseline $\left(\mathrm{kg} / \mathrm{m}^{2}\right)(\mathrm{SD})$ & $28.3(6.2)$ & $28.0(5.8)$ & $28.2(6.0)$ & $27.5(5.1)$ \\
\hline
\end{tabular}

with three or more measures (Table 4). This indicates that persons with incomplete data were not generally on steeper BMI trajectories than those with complete data.

\section{Discussion}

Consistent with prior work in the U.S. and other industrialized countries $(19,20)$, we found an inverse cross-sectional association between socioeconomic position (as assessed by income or education) and BMI in white women. Also consistent with prior work (19-21), education and income were inversely associated with BMI in black women. We also found inverse, although weak, associations of education and income with BMI in white men. In contrast, income and education were positively associated with BMI in black men. Prior studies have also found evidence of sex differences in the socioeconomic patterning of BMI. Although some studies have reported inverse associations of socioeconomic indicators with BMI in white men (20), others have not $(20,22)$. The positive association between socioeconomic position and BMI in U.S. black men has also been previously reported $(19,21)$. Our results regarding the socioeconomic patterning of BMI in white and black men and women are also consistent with those recently reported by Zhang and Wang (8) using National Health and Nutrition Examination Study III data, which was collected close to the period of the ARIC baseline exam (1987 to 1989), on which our cross-sectional analyses are based. The reasons for these race and sex differences in the social patterning of BMI remain to be determined.

Neighborhood characteristics have been infrequently investigated in relation to BMI. Several years ago, a British study comparing four contrasting neighborhoods found that neighborhood deprivation was associated with greater BMI after controlling for age, sex, and social class (23). We found that neighborhood disadvantage was associated with greater BMI in women but not in men. The only other large-scale study of neighborhood characteristics and BMI in the U.S. also found community disadvantage to be independently and positively associated with BMI in women but not in men (24).

As in other recent studies (25-28), BMI increased over time in almost all age groups. However, contrary to expectation, BMI increases over time were not greater in the lower socioeconomic groups. Mean BMI increased over the follow-up for all education, income, and neighborhood cat- 


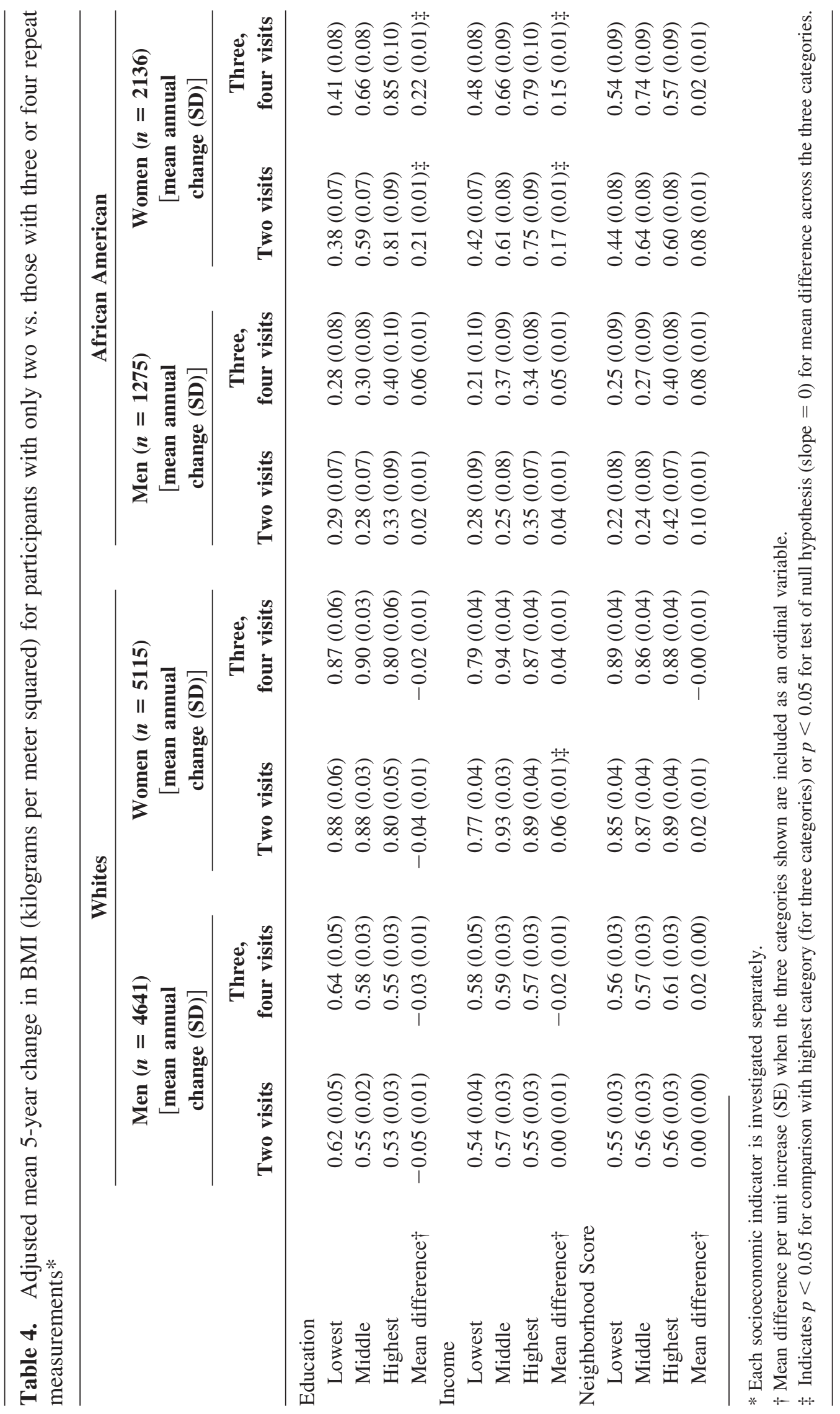


egories. There was no evidence of greater increases over time in the lower SES groups. In fact, in black men and women, there was some evidence that BMI increases were actually greater in the higher than in the lower socioeconomic groups. Adjustment for variables associated with both BMI and socioeconomic factors such as smoking, self-reported health, and history of cancer as time-dependent covariates did not modify these results.

Studies of changes in BMI over time by socioeconomic factors have not always reported consistent results. A study of adult men ages 25 to 44 years found greater increases in BMI over a 10 -year period for men with 12 or fewer years of education compared with more educated men (29). Another study of young adults ages 18 to 30 years also found greater increases in BMI over a 5-year period in less educated individuals (30). In children, adiposity has been reported to increase more rapidly in lower SES than in higher SES youth $(30,31)$. In contrast, another study of young adults (18 to 30 years old) living in the U.S. found no significant differences in weight changes over a 10-year period by education (25). Studies conducted in Europe also failed to find differences in longitudinal trends in BMI by education, occupation, or income in adults $(27,28)$, with the exception of one study in Poland that found greater increases in weight in lower educated women (32). To our knowledge, no studies have examined longitudinal changes in BMI by neighborhood characteristics.

Our results regarding longitudinal trends by SES are consistent with the changes in socioeconomic differentials over time reported by Zhang and Wang based on the analyses of nationally representative repeat cross-sectional surveys in the U.S. (National Health and Nutrition Examination Study data) (8). Zhang and Wang found that the inverse associations of education with obesity and BMI observed in white and black men and women in the 1970s had generally been reduced or had even disappeared by the late 1990s. This pattern is consistent with similar increases in obesity over time across SES groups or with greater increases in the higher SES groups.

There are at least two methodological reasons that could have limited our ability to detect socioeconomic or neighborhood differences in changes over time in BMI. If persons lost to follow-up are selected on the basis of both baseline socioeconomic indicators and BMI trajectories (with more rapidly increasing BMI in those lost to follow-up), the analytic methods we used (which assume missing at random) could have resulted in underestimates of trajectory differences by SES (33). Thus, if persons with a smaller number of BMI measures (due to death or loss to follow-up) were also more likely to have increasing BMI over time, we may have underestimated socioeconomic differences in BMI change. Although loss to follow-up was clearly associated with lower socioeconomic position at baseline, there was no evidence that person with fewer visits were on steeper BMI trajectories than those with three or four visits.

Existing socioeconomic differences in baseline BMI could also have limited our ability to detect SES differences in trajectories. Persons who already have very high BMI at baseline may be unlikely to increase even more over time. In an attempt to control for the underlying effects of baseline BMI, we performed stratified analyses to create more homogeneous subsets of participants on the basis of baseline BMI. We divided our sample into three strata (BMI < 25 , BMI 25 to 29.9, BMI $\geq 30$ ). Although increases were greater in persons with lower BMI at baseline, no clear patterns in changes in BMI over time by SES emerged within baseline BMI strata (data not shown). Although the two approaches we used (comparing BMI change in persons with more complete and less complete data and stratifying by BMI) do not completely eliminate the possibility that these two factors hampered our ability to detect socioeconomic differences, they do suggest that their effects are probably not substantial.

Because of the limited overlap in socioeconomic indicators, race-specific categories were used. This ensures sufficient sample size in each group but has the disadvantage that categories are not comparable across race-groups, making it impossible to draw inferences regarding race differences at similar levels of income or neighborhood characteristics. We repeated the analyses using similar income and neighborhood categories in both race groups (data not shown) and found qualitatively similar results, although SEs were large in some categories due to limited sample size.

Two important limitations of our study in the investigation of neighborhood differences are the use of census block groups as proxies for neighborhoods and the absence of direct measurements of the specific features of neighborhoods potentially relevant to weight change. Examples of relevant features include characteristics of the built environment conducive to walking or physical activity, availability and price of healthy foods, and advertising for unhealthy foods. Differences across neighborhoods in these attributes may have been poorly captured by the neighborhood socioeconomic score. This misspecification of neighborhoods and their relevant attributes may have seriously hampered our ability to detect neighborhood effects on weight change. We also did not examine the impact of change in neighborhood of residence on weight, which may be the more relevant question from an intervention point of view. Preliminary data from the Moving to Opportunity project, the only randomized trial of neighborhood health effects to date, suggest that moving from a poor to a non-poor neighborhood results in decreases in BMI in adults (34).

The ARIC sample comprises a random population-based sample of middle-aged adults living in four different regions of the U.S. It is not intended to be a nationally representa- 
tive sample; therefore, estimates of mean BMI or prevalence of obesity are clearly not generalizable to the full U.S. population. However, there is no a priori reason to believe that our estimates of the associations between socioeconomic indicators and BMI would have been very different in a nationally representative sample. Our results are also consistent with prior studies of other samples $(19-21,24,25)$ as well as with analyses of nationally representative samples (8). A large proportion of the sample was close to retirement age at baseline. Income is clearly limited as a measure of socioeconomic position in the elderly (35). Education was used as an alternative indicator with generally consistent results. Although we controlled for a set of time-dependent covariates (smoking, cancer, and self-reported health), which could confound associations between SES and changes in BMI over time, it is possible that residual confounding contributed to our inability to detect SES differences in trends over time. It is also possible that 9 years of follow-up was not sufficient to detect differences in trends over time. Finally, because a large proportion of black participants were sampled from only one site, race differences observed in the ARIC cohort may be confounded by region of residence and may not be generalizable to blackwhite differences in the U.S. generally.

In this middle-aged sample, recent increases in BMI seem to have occurred similarly across socioeconomic groups, suggesting that factors affecting the population as a whole are likely to be involved. If confirmed, these findings suggest that broad, population-wide strategies are needed to control the obesity epidemic. However, we did document important BMI differences at baseline, with greater BMI in socioeconomically disadvantaged groups in white and black women. These socioeconomic differences in BMI probably emerged over childhood and adolescence, much earlier than the baseline exam of our cohort, and possibly at a time when socioeconomic factors were more strongly and inversely associated with BMI in most race and sex groups (8). The reasons for the positive associations of socioeconomic factors with BMI in black men and the positive associations of SES with BMI increases in black women and men are worthy of additional investigation.

\section{Acknowledgments}

The ARIC Study is carried out as a collaborative study supported by National Heart, Lung, and Blood Institute Contracts N01-HC-55015, N01-HC-55016, N01-HC55018, N01-HC-55019, N01-HC-55020, N01-HC-55021, and N01-HC-55022. We thank the staff and participants of the ARIC study for their important contributions and Dr. Paul Sorlie for his constructive suggestions in reviewing this manuscript. This work was funded, in part, by the National Heart, Lung, and Blood Institute, by the NIH Grant R01HL64142, and by the Columbia Center for Health of
Urban Minorities (MD00206) from The National Center on Minority Health Disparities, National Institutes of Health.

\section{References}

1. Manson JE, Bassuk SS. Obesity in the United States: a fresh look at its high toll. JAMA. 2003;289:229-30.

2. Flegal KM, Carroll MD, Kuczmarski RJ, Johnson CL. Overweight and obesity in the United States: prevalence and trends, 1960-1994. Int J Obes Relat Metab Disord. 1998;22: $39-47$.

3. Ogden CL, Carroll MD, Flegal KM. Epidemiologic trends in overweight and obesity. Endocrinol Metab Clin North Am. 2003;32:741-60, vii.

4. Kuczmarski RJ, Flegal KM, Campbell SM, Johnson CL. Increasing prevalence of overweight among US adults: The National Health and Nutrition Examination Surveys, 1960 to 1991. JAMA. 1994;272:205-11.

5. Mokdad AH, Serdula MK, Dietz WH, Bowman BA, Marks JS, Koplan JP. The spread of the obesity epidemic in the United States, 1991-1998. JAMA. 1999;282:1519-22.

6. Flegal KM, Carroll MD, Ogden CL, Johnson CL. Prevalence and trends in obesity among US adults, 1999-2000. JAMA. 2002;288:1723-7.

7. Mokdad AH, Bowman BA, Ford ES, Vinicor F, Marks JS, Koplan JP. The continuing epidemics of obesity and diabetes in the United States. JAMA. 2001;286:1195-200.

8. Zhang Q, Wang Y. Trends in the association between obesity and socioeconomic status in U.S. adults: 1971 to 2000. Obes Res. 2004;12:1622-32.

9. Kaplan GA, Keil JE. Socioeconomic factors and cardiovascular disease: a review of the literature. Circulation. 1993;88: 1973-98.

10. Diez Roux AV, Merkin SS, Arnett D, et al. Neighborhood of residence and incidence of coronary heart disease. $N$ Engl $J$ Med. 2001;345:99-106.

11. Sundquist K, Winkleby M, Ahlen H, Johansson SE. Neighborhood socioeconomic environment and incidence of coronary heart disease: a follow-up study of 25,319 women and men in Sweden. Am J Epidemiol. 2004;159:655-62.

12. Diez Roux AV. Residential environments and cardiovascular risk. J Urban Health. 2003;80:569-89.

13. Steptoe A, Marmot M. The role of psychobiological pathways in socio-economic inequalities in cardiovascular disease risk. Eur Heart J. 2002;23:13-25.

14. Investigators TA. The Atherosclerosis Risk in Communities (ARIC) Study: design and objectives. Am J Epidemiol. 1989; 129:687-702.

15. Diez-Roux AV, Kiefe CI, Jacobs DR, et al. Area characteristics and individual-level socioeconomic position indicators in three population-based epidemiologic studies. Ann Epidemiol. 2001;11:395-405.

16. Bureau of the Census. 1980 Census of Population: Classified Index of Industries and Occupations. Washington DC: Government Printing Office; 1982

17. Jacobs DR Jr, Hannan PJ, Wallace D, Liu K, Williams OD, Lewis CE. Interpreting age, period and cohort effects in plasma lipids and serum insulin using repeated measures regression analysis: the CARDIA Study. Stat Med. 1999;18: $655-79$. 
18. Juhaeri, Stevens J, Jones DW, Arnett D. Associations of aging and birth cohort with body mass index in a biethnic cohort. Obes Res. 2003;11:426-33.

19. Burke GL, Savage PJ, Manolio TA, et al. Correlates of obesity in young black and white women: the CARDIA Study. Am J Public Health. 1992;82:1621-5.

20. Sobal J, Stunkard AJ. Socioeconomic status and obesity: a review of the literature. Psychol Bull. 1989;105:260-75.

21. Croft JB, Strogatz DS, Keenan NL, James SA, Malarcher AM, Garrett JM. The independent effects of obesity and body fat distribution on blood pressure in black adults: the Pitt County study. Int J Obes Relat Metab Disord. 1993;17:391-7.

22. Luepker RV, Rosamond WD, Murphy R, et al. Socioeconomic status and coronary heart disease risk factor trends: The Minnesota Heart Survey. Circulation. 1993;88:2172-9.

23. Ellaway A, Anderson A, Macintyre S. Does area of residence affect body size and shape? Int J Obes Relat Metab Disord. 1997;21:304-8.

24. Robert SA, Reither EN. A multilevel analysis of race, community disadvantage, and body mass index among adults in the US. Soc Sci Med. 2004;59:2421-34.

25. Lewis CE, et al. Weight gain continues in the 1990s: 10-year trends in weight and overweight from the CARDIA study: Coronary Artery Risk Development in Young Adults. Am J Epidemiol. 2000;151:1172-81.

26. Lahti-Koski M, Jousilahti P, Pietinen P. Secular trends in body mass index by birth cohort in eastern Finland from 1972 to 1997. Int J Obes Relat Metab Disord. 2001;25:727-34.

27. van Lenthe FJ, Droomers M, Schrijvers CT, Mackenbach JP. Socio-demographic variables and 6 year change in body mass index: longitudinal results from the GLOBE study. Int $J$ Obes Relat Metab Disord. 2000;24:1077-84.

28. Sundquist J, Johansson SE. The influence of socioeconomic status, ethnicity and lifestyle on body mass index in a longitudinal study. Int J Epidemiol. 1998;27:57-63.

29. Kahn HS, Williamson DF. The contributions of income, education and changing marital status to weight change among US men. Int J Obes. 1990;14:1057-68.

30. Burke GL, Bild DE, Hilner JE, Folsom AR, Wagenknecht LE, Sidney S. Differences in weight gain in relation to race, gender, age and education in young adults: the CARDIA Study: Coronary Artery Risk Development in Young Adults. Ethn Health. 1996;1:327-35.

31. Moore DB, Howell PB, Treiber FA. Changes in overweight in youth over a period of 7 years: impact of ethnicity, gender and socioeconomic status. Ethn Dis. 2002;12:S1-83-6.

32. Dennis BH, Pajak A, Pardo B, Davis CE, Williams OD, Piotrowski W. Weight gain and its correlates in Poland between 1983 and 1993. Int J Obes Relat Metab Disord. 2000; 24:1507-13.

33. Little RJ, Raghunathan T. On summary measures analysis of the linear mixed effects model for repeated measures when data are not missing completely at random. Stat Med. 1999; 18:2465-78.

34. Orr L. Moving to Opportunity for Fair Housing Demonstration: Interim Impacts Evaluation. Washington, DC: US Department of Housing and Urban Development, Office of Policy Development and Research; 2003.

35. Berkman LF, Macintyre S. The measurement of social class in health studies: old measures and new formulations. IARC Sci Publ. 1997:51-64. 